\title{
Selective Serotonin Reuptake Inhibitors, Serotonin-Norepinephrine Reuptake Inhibitors and Migraine: Should Cochrane Close the Door?
}

\section{Daniel Shevel}

Medical Research Institute, Cape Town, South Africa

Correspondence to: Daniel Shevel, Danielceo@m-r-i.net

Keywords: Migraine, ICHD, SSRI, Depression, Cochrane

Received: March 9, $2018 \quad$ Accepted: April 23, 2018

Published: April 26, 2018

Copyright $\odot 2018$ by author and Scientific Research Publishing Inc.

This work is licensed under the Creative Commons Attribution International License (CC BY 4.0).

http://creativecommons.org/licenses/by/4.0/

\section{(c) (1) Open Access}

\section{ABSTRACT}

The Cochrane Collaboration completed a review entitled "Selective Serotonin Reuptake Inhibitors (SSRIs) and Serotonin-norepinephrine Reuptake Inhibitors (SNRIs) for the Prevention of Migraine in Adults" in 2015. In the review, they acknowledge that poor-quality research and low levels of evidence make it difficult to draw definitive conclusions, but then state that "A randomised controlled trial comparing a SSRI or a SNRI versus another drug or another non-pharmacological intervention is not a priority in the migraine research pipeline and might not exert a significant impact on the overall evidence". A detailed evaluation of 10 of the 11 papers analysed in the Cochrane Review reveals that 9 of them use the International Classification of Headache Disorders (ICHD) system to determine their study cohorts. Despite being the current globally accepted standard for classification, diagnosis and categorization of migraine, the ICHD criteria are based on an arbitrary symptom list and offer little, if any, insight into the possible mechanisms involved in the pathophysiology of a migraine. Using the ICHD system in scientific studies which investigate the prevention and treatment of migraines, including those analyzed by the 2015 Cochrane Review, therefore results in amorphous cohort selection for those studies. This directly impacts the clinical relevance of the results drawn from the research. We acknowledge, and are grateful for, the important and highly credible contribution that the Cochrane Collaboration brings to our body of scientific knowledge. We are, however, concerned that the conclusions drawn in this important review might negatively impact on clinician ability to help individual migraine patients, not through any fault of the Cochrane Collaboration review system, but due to a fundamental flaw in the classification of migraines. 


\section{INTRODUCTION}

The Cochrane Collaboration recently completed a review entitled "Selective Serotonin Reuptake Inhibitors (SSRIs) and Serotonin-norepinephrine Reuptake Inhibitors (SNRIs) for the Prevention of Migraine in Adults" [1]. The reviewers acknowledge that poor-quality research and low levels of evidence have affected their results.

We suggest that fundamental migraine-classification issues, inherent in the International Classification of Headache Disorders (ICHD) criteria, have also played a significant role in their findings. We therefore question their conclusion that "A randomised controlled trial comparing a SSRI or a SNRI versus another drug or another non-pharmacological intervention is not a priority in the migraine research pipeline and might not exert a significant impact on the overall evidence" [1].

\section{HISTORY}

Debate and discussion regarding both the pathophysiology of migraine and its treatment is on-going. Many of the currently used prophylactic migraine medications are:

1) discovered by accident during an individual's treatment for a co-existing condition.

2) used simply because their earlier counterparts have shown some success.

The precursors to SSRIs and SNRIs, tricyclic antidepressants, are well-established as treatment in the prophylaxis of migraine. As a result, in a clinical setting, SSRIs and SNRIs are often recommended [2-4]. However, no consensus on their use and effectiveness in the prophylactic treatment of migraine currently exists.

Table 1 illustrates how varied the current migraine-treatment guidelines for SSRIs and SNRIs are, even amongst well-recognized international bodies in the field. It is clear that clinical practitioners face

Table 1. Current global guidelines for the use of selective serotonin reuptake inhibitors and serotonin-norepinephrine reuptake inhibitors in the prophylactic treatment of migraine ${ }^{a}$.

\begin{tabular}{cl}
\hline Organization & \multicolumn{1}{c}{ Current Recommendation } \\
\hline & "Recommended agents based on consensus and clinical experience: \\
& cyproheptadine, bupropion, diltiazem, doxepin, fluvoxamine \\
US Headache Consortium & $\begin{array}{l}\text { ibuprofen, imipramine, mirtazepine, nortriptyline, paroxetine } \\
\text { protriptyline, sertraline }\end{array}$ \\
& {$[5]$}
\end{tabular}

American Academy of Family

Physicians and the American

College of

Physicians-American

Society of Internal Medicine

(ACP-ASIM)

No recommendation made about the use of SSRIs and/or SNRIs for the preventative treatment of migraine [5]

American Academy of Neurology (AAN) and the American Headache Society (AHS)
Anti-depressants, SSRIs, SNRIs, tricyclic antidepressants [TCAs]and venlafaxine $^{\mathrm{b}}$ are classified as Level B: medications are "probably effective" (one class 1 or two class 2 studies) [6]

Fluvoxamine $^{\mathrm{b}}$ and fluoxetine $\mathrm{e}^{\mathrm{b}}$, i.e. two specific SSRIs, are classified as Level U: "inadequate or conflicting data to support or refute use" [6] 


\section{Continued}

British Association for the Study of Headache (BASH)

Canadian Headache Society (CHS)

European Federation of Neurological Sciences (EFNS)
Classified as a third-line prophylactic treatment. "SSRIs are of uncertain value. Fluoxetine ${ }^{\mathrm{b}} 20 \mathrm{mg}$ alter die to $40 \mathrm{mg}$ od is best studied with inconclusive evidence of efficacy against migraine" [7]

Venlafaxine $e^{\mathrm{b}}$ is classified as a "weak recommendation, low quality evidence... We recommend that clinicians offer venlafaxine ${ }^{\mathrm{b}}$ extended release at a target dose of $150 \mathrm{mg}$ per day to eligible patients for migraine prophylaxes" [8]

"For femoxetine $e^{\mathrm{b}}$, two small, positive placebo controlled trials have been published" [9]

"Fluoxetine $e^{\mathrm{b}}$ in doses between 10 and $40 \mathrm{mg}$ was effective in three and not effective in one placebo controlled trial" [9]

"Venlafaxine $e^{\mathrm{b}}$ extended release (dose 75 - $150 \mathrm{mg}$ ) has shown efficacy in one placebo controlled and two open trials and can therefore be recommended as a second-choice anti-depressant in migraine prophylaxis (second to amitriptyline)"

aPlease note: In some cases, for the benefit of the reader, the guidelines' original wording has been paraphrased. ${ }^{b}$ Fluvoxamine, paroxetine, sertraline, fluoxetine andfemoxetine are SSRIs; venlafaxine is an SNRI.

difficult choices as they seek to give their patients appropriate advice about the use of SSRIs and SNRIs in the prevention of migraine.

It follows that such an important topic warranted comprehensive review and this was undertaken by the Cochrane Collaboration in 2005 and updated by them in 2015.

\section{2015 COCHRANE COLLABORATION META-ANALYSIS OF MIGRAINE-PREVENTION TREATMENT}

Any Cochrane Collaboration review is considered to be an important contribution to the collective understanding of current medical research. They are enormously respected around the world for their scientifically sound processes, careful and detailed analysis of methodologies, classification of evidence levels and assessment of bias when conducting a systematic review. Their findings are therefore likely to significantly impact practitioners' clinical decision making.

The Cochrane Collaboration conducted an initial review of the use of SSRIs and SNRIs in migraine and tension-type headache prophylaxis in 2005 [10]. Then, in April 2015, they updated that systematic review with a report entitled "Selective Serotonin Reuptake Inhibitors (SSRIs) and Serotonin-norepinephrine Reuptake Inhibitors (SNRIs) for the Prevention of Migraine in Adults" [1], hereafter referred to as the 2015 Cochrane Review.

In contrast to the 2005 review, the 2015 Cochrane Review separates tension-type headaches (TTHs) from migraines. A separate review on the use of SSRIs and SNRIs for the prevention of TTHs was published later, in May 2015 [11]. This article focuses only on the prevention-of-migraine review and not on the TTH review.

The 2015 Cochrane Review involved a meta-analysis of 11 studies. One of those [12], written in Italian, we are currently unable to trace. Table 2 summarizes the conclusions drawn by the remaining 10 studies' authors. 
Table 2. Conclusions drawn by the authors of 10 of the 11 papers reviewed in the 2015 Cochrane Collaboration meta-analysis, "Selective Serotonin Reuptake Inhibitors (SSRIs) and Serotonin-norepinephrine Reuptake Inhibitors (SNRIs) for the Prevention of Migraine in Adults" [1] c.

\begin{tabular}{cl}
\hline Study Author & Study Conclusion \\
\hline
\end{tabular}

Bank, 1994: 476 [14]

Bulut et al., 2004: 44 [2]

"The beneficial effects [of fluoxetine] observed in this study, combined

Adly et al., 1992: 103 [13]

with the relative lack of significant side effects, should encourage others

to try to replicate these findings with a larger group over a longer period

of observation"

"These findings suggest, that fluvoxamine (vs. amitriptyline) may be an alternative drug in migraine prophylaxis, however, further studies should be performed with more subjects"

"In conclusion, it is suggested that venlafaxine may be considered for the prophylaxis of migraine because of its low and/or tolerable side effect

properties"

NOTE: Financially supported by a pharmaceutical company producing venlafaxine

"Even if preliminary and to be confirmed in a larger number of patients,

Colucci d'Amato et al., 1999: 718-719 [15]

Krymchantowski et al., 2002: $513[16]$

Landy et al., 1999: 31 [17]

Oguzhanoglu et al., 1999: 532

[18]

Ozyalcin et al., 2005: 144 [19] these data seem to support the use of fluoxetine in migraine prophylaxis, especially when conventional treatments give no results or have serious side effects limiting their use"

"We could not demonstrate superiority for the combination of amitriptyline and fluoxetine over amitriptyline alone in treating transformed migraine"

“This present study and the previous literature on selective serotonin reuptake inhibitors for migraine prophylaxis do not support a major role for the use of this class of medication in migraine"

NOTE: Supported by a charity organization

"Both drugs [amitriptyline and fluoxetine] benefited chronic tension-type headache and episodic tension-type headache although fluoxetine effects were less sustained. Again, increasing the dose of FLX [fluoxetine] might have provided better effect"

"Venlafaxine was more effective than placebo and is safe and well tolerated as migraine prophylaxis"

NOTE: Financially supported by a pharmaceutical company producing venlafaxine 
Steiner et al., 1998: 286 [3]

Tarlaci, 2009: 254 [4]
"S-fluoxetine, the long acting S-enantiomer of fluoxetine, is worth more formal evaluation in migraine prophylaxis, notwithstanding that (or perhaps especially because) racemic fluoxetine may be inactive" NOTE: Supported by a pharmaceutical company producing fluoxetine and planned as a phase II study which would be followed by a pivotal phase III study if the outcome was encouraging

"According to our findings, venlafaxine and escitalopram are both effective in the prophylaxis of migraine without depression and anxiety. This effect was independent of mood disorder"

'Please note: In some cases, for the benefit of the reader, the guidelines' original wording has been paraphrased.

When viewed collectively, the conclusions are confusing and sometimes contradictory.

\section{RESEARCH QUALITY CONCERNS (AS HIGHLIGHTED BY THE COCHRANE COLLABORATION)}

The 2015 Cochrane Review carefully analyses the potential bias of each study covered by its meta-analysis. It also details the many and varied weaknesses of the individual studies.

With reference to "Overall completeness and applicability of evidence", the review concludes that: "The data that inform this review are few and generally poor, in terms of the quality of the trials that originated them. Only five studies reported data on the most relevant clinical outcome, migraine frequency (two placebo and three amitriptyline-controlled) for a total of fewer than 300 participants. Reporting was often incomplete, making some studies uninformative. The applicability of this scarce evidence is also an issue, mainly because the analyzed studies used short follow-up and outcomes with a small clinical value. However, the findings of this review suggest that SSRIs and SNRIs do not show benefits for the outcomes that may matter to patients" [1].

Without doubt, it is difficult to draw definitive, trustworthy conclusions from this data.

\section{FURTHER COMPLICATIONS WITH SAMPLE SELECTION, NOT REPORTED BY COCHRANE}

The current globally accepted standard for classification, diagnosis and categorization of migraine is the International Classification of Headache Disorders (ICHD) criteria [20]. The 1st edition of the ICHD was released by the International Headache Society (IHS) in 1988 and its preface made the recommendation that this classification should be put to "immediate use in scientific studies" [21]. The 2nd edition, published in 2004, stated that "No journal should publish papers related to headache that are not using this classification and the associated diagnostic criteria" [22]. This classification system has therefore become the norm for classification of migraine in scientific, published studies. It is rare indeed to find a published study which does not use it when selecting study cohorts.

But the IHS/ICHD is a controversial system which may well have introduced fundamental flaws into almost all of the studies the Cochrane Collaboration analyzed in 2015.

A 2001 edition of "Headache" quotes Professor Egilius L.H. Spierings (neurologist, clinical professor, Director of the Headache and Facial Pain Program at Tufts University, previously Department of Neurology, Harvard Medical School, and past editor of "Cephalgia"): "The IHS classification lacks biologi- 
$\mathrm{cal} /$ clinical validity and therefore undermines advancement in our understanding of headache. It is a waste of time, money and effort and potentially misleading to study from a biological/clinical perspective a condition which, as in the classification, is defined purely on arbitrary grounds" [23].

Professor Jes Oleson has been the Chairman of the Headache Classification Committee of the IHS since its inception 35 years ago. Prof. Oleson states in a paper he co-authored in 2001: "The IHS criteria were developed without the collection of empiric data" [24] and "The IHS criteria were based on opinion" [24].

The IHS/ICHD system uses a combination of criteria to diagnose a migraine (Table 3 ).

A paper published in 2014 provides detailed analysis of each criteria used for the diagnosis of migraine according to the IHS/ICHD-II classification system [26]. It concludes that no supporting data were supplied by the IHS/ICHD for any of the following criteria used to diagnose migraine: number of attacks, duration of attacks, pain intensity, aggravation of pain with mild exercise, photophobia, phonophobia or nausea and vomiting. For criteria where data did exist, i.e. unilateral pain and pulsatile pain, data provided in IHS/ICHD-II [27-29] diametrically contradict the inclusion of these variables as diagnostic criteria for migraine. When abeta version of IHS/ICHD-3was released in 2013 [25], these contradictory data had been removed from the references, but no changes to the structure of the classification had been made.

On the IHS/ICHD-3beta website (http://beta.ichd-3.org/), Prof. Oleson stated that "For this [3rd] edition, there has been a substantial body of evidence available for the classification work, in contrast to our previous editions, which were mostly based on the opinions of experts. We have tried to be conservative, making changes only where there was good published evidence to support change or where the need for change was intuitively obvious" [25]. But careful analysis of IHS/ICHD-3beta did not provide this body of evidence supporting the classification system.

A direct written request was made to Prof. Oleson in 2014 (see Appendix A) asking for:

1) the body of evidence referred to in IHS/ICHD-3beta.

2) an explanation for why an important article referenced in IHS/ICHD-II, which contains data contradicting the inclusion of unilateral or pulsating headache as criteria for migraine [29], has been removed from IHS/ICHD-3beta.

3) an explanation for why an important article referenced in IHS/ICHD-II, in which Prof. Oleson states that "The IHS criteria were developed without the collection of empirical data" [24] and "The IHS criteria were based on opinions" [24], has been removed from IHS/ICHD-3beta.

Table 3. IHS/ICHD (versions 1-3) criteria for diagnosis of migraine [20, 25].

\begin{tabular}{ll}
\hline A & Criteria \\
B & Headache attacks lasting $4-72 \mathrm{hr}$ (untreated or unsuccessfully treated) \\
& Headache has at least two of the following four characteristics: \\
1) unilateral location \\
2) pulsating quality \\
3) moderate or severe pain intensity \\
4) aggravation by or causing avoidance of routine physical activity (eg, walking or climb- \\
ing stairs) \\
D & $\begin{array}{l}\text { During headache at least one of the following: } \\
\text { E }\end{array}$ \\
& 2) photophobia and phonophobia \\
\end{tabular}


4) confirmation that the migraine-classification criteria in IHS/ICHD-3beta still appear to be based on unsubstantiated opinions and a request to please provide evidence to the contrary if such evidence exists.

Four years later, in 2018, this request remains unanswered.

As this paper went to publication in early 2018, the finalized 3rd edition of the IHS/ICHD classification system [20] was released. Unfortunately, it maintains its predecessors' fundamental flaws.

\section{CONCLUSIONS}

The pathophysiology behind migraine is not yet fully understood so the assumption that one pathophysiological pathway exists for all migraine sufferers is a clinically limiting and disease-centered approach. This "all migraines have the same pathophysiology" mindset complicates effective research and clinical interpretation of results.

Only one of the 2015 Cochrane Review studies we evaluated [13] did not use IHS/ICHD as their inclusion criteria and none made a distinction between the possible pathophysiological mechanisms behind their patients' migraines.

Based on an arbitrary symptom list, this current classification system offers little, if any, insight into the possible mechanisms involved in the pathophysiology of a migraine. Using it in scientific studies investigating the prevention and treatment of migraines, such as those analyzed by the 2015 Cochrane Review, therefore leads to amorphous cohort selection for those studies. This directly impacts the clinical relevance of the results drawn from the research.

While the 2015 Cochrane Review is sound and conducted according to the Cochrane Collaboration's usual high standards, Cochrane conclude their review with the following statement: "However, overall we think that the value of new studies comparing anti-depressants in this setting is questionable. A randomized controlled trial comparing a SSRI or a SNRI versus another drug or another non-pharmacological intervention is not a priority in the migraine research pipeline and might not exert a significant impact on the overall evidence" [1].

We would argue that amorphous, arbitrary, symptomatic selection of cohorts complicates interpretation of data. In the meantime, the Cochrane Collaboration's conclusions are likely to be impacting clinical guidelines around the world.

Future migraine research should carefully consider cohort selection, based on the possible pathophysiology of each patient's migraine, and should consider the ample muscular and arterial data available when classifying migraine patients. Continued use of the ICHD classification system is likely to hinder development in this important area and delay effective treatment for millions of migraine sufferers.

\section{REFERENCES}

1. Banzi, R., Cusi, C., Randazzo, C., Sterzi, R., Tedesco, D. and Moja, L. (2015) Selective Serotonin Reuptake Inhibitors (SSRIs) and Serotonin-Norepinephrine Reuptake Inhibitors (SNRIs) for the Prevention of Migraine in Adults.

http://www.cochrane.org/CD002919/SYMPT_selective-serotonin-reuptake-inhibitors-ssris-and-serotonin-nore pinephrine-reuptake-inhibitors-snris

2. Bulut, S., Berilgen, M.S., Baran, A., Tekatas, A., Atmaca, M. and Mungen, B. (2004) Venlafaxine Versus Amitriptyline in the Prophylactic Treatment of Migraine: Randomized, Doubleblind, Crossover Study. Clinical Neurology and Neurosurgery, 107, 44-48. https://doi.org/10.1016/j.clineuro.2004.03.004

3. Steiner, T.J., Ahmed, F., Findley, L.J., MacGregor, E.A. and Wilkinson, M. (1998) S-Fluoxetine in the Prophylaxis of Migraine: A Phase II Double-blind Randomized Placebo-Controlled Study. Cephalalgia, 18, 283-286. https://doi.org/10.1046/j.1468-2982.1998.1805283.x

4. Tarlaci, S. (2009) Escitalopram and Venlafaxine for the Prophylaxis of Migraine Headache without Mood Dis- 
orders. Clinical Neuropharmacology, 32, 254-258. https://doi.org/10.1097/WNF.0b013e3181a8c84f

5. Schroeder, B.M. (2003) AAFP/ACP-ASIM Release Guidelines on the Management and Prevention of Migraines. The American Academy of Family Physicians. http://www.aafp.org/afp/2003/0315/p1392.html\#

6. Silberstein, S.D., Holland, S., Freitag, F., Dodick, D.W., Argoff, C. and Ashman, E. (2012) Evidence-Based Guideline Update: Pharmacologic Treatment for Episodic Migraine Prevention in Adults. Neurology, 78, 1337-1345. https://doi.org/10.1212/WNL.0b013e3182535d20

7. MacGregor, E.A., Steiner, T.J. and Davies, P.T.G. (2010) Guidelines for All Healthcare Professionals in the Diagnosis and Management of Migraine, Tension-Type Headache, Cluster Headache and Medication-overuse Headache. British Association for the Study of Headache. 3rd Edition (1st Revision), 1-53.

8. Pringsheim, T., Davenport, W.J. and Mackie, G. (2012) Canadian Headache Society Guideline for Migraine Prophylaxis. Canadian Journal of Neurological Sciences, 39, 17.

9. Evers, S., Afra, J., Frese, A., Goadsby, P.J., Linde, M., May, A. and Sándor, P.S. European Federation of Neurological Societies (2009) EFNS Guideline on the Drug Treatment of Migraine-Revised Report of an EFNS Task Force. European Journal of Neurology, 16, 968-981. https://doi.org/10.1111/j.1468-1331.2009.02748.x

10. Moja, L., Cusi, C., Sterzi, R.R. and Canepari, C. (2005) Selective Serotonin Re-uptake Inhibitors (SSRIs) for Preventing Migraine and Tension-type Headaches. http://onlinelibrary.wiley.com/doi/10.1002/14651858.CD002919.pub2/full

11. Banzi, R., Cusi, C., Randazzo, C., Sterzi, R., Tedesco, D. and Moja, L. (2015b) Selective Serotonin Reuptake Inhibitors (SSRIs) and Serotonin-norepinephrine Reuptake Inhibitors (SNRIs) for Preventing Tension-type Headache.

www.cochrane.org/CD011681/SYMPT_selective-serotonin-reuptake-inhibitors-ssris-and-serotonin-norepineph rine-reuptake-inhibitors-snris

12. Polisca, R., Signoretti, P. and Marchi, P. (1992) Fluoxetine in the Treatment of Migraine with Interval Headache [La fluoxetina nella terapia della emicrania con cefalea intervallare]. Rassegna Internazionale di Clinica e Terapia, 72, 408-415.

13. Adly, C., Straumanis, J. and Chesson, A. (1992) Fluoxetine Prophylaxis of Migraine. Headache, 32, 101-104. https://doi.org/10.1111/j.1526-4610.1992.hed3202101.x

14. Bank, J. (1994) A Comparative Study of Amitriptyline and Fluvoxamine in Migraine Prophylaxis. Headache, 34, 476-478. https://doi.org/10.1111/j.1526-4610.1994.hed3408476.x

15. Colucci d'Amato, C., Pizza, V., Marmolo, T., Giordano, E., Alfano, V. and Nasta, A. (1999) Fluoxetine for Migraine Prophylaxis: ADouble-blind Trial. Headache, 39, 716-719.

https://doi.org/10.1046/j.1526-4610.1999.3910716.x

16. Krymchantowski, A.V., Silva, M.T., Barbosa, J.S. and Alves, L.A. (2002) Amitriptyline versus Amitriptyline Combined with Fluoxetine in the Preventative Treatment of Transformed Migraine: A Double-Blind Study. Headache, 42, 510-514. https://doi.org/10.1046/j.1526-4610.2002.02125.x

17. Landy, S., McGinnis, J., Curlin, D. and Laizure, S.C. (1999) Selective Serotonin Reuptake Inhibitors for Migraine Prophylaxis. Headache, 39, 28-32. https://doi.org/10.1046/j.1526-4610.1999.3901028.x

18. Oguzhanoglu, A., Sahiner, T., Kurt, T. and Akalin, O. (1999) Use of Amitriptyline and Fluoxetine in Prophylaxis of Migraine and Tension-Type Headaches. Cephalalgia, 19, 531-532.

https://doi.org/10.1046/j.1468-2982.1999.019005531.x

19. Ozyalcin, S.N., Talu, G.K., Kiziltan, E., Yucel, B., Ertas, M. and Disci, R. (2005) The Efficacy and Safety of Venlafaxine in the Prophylaxis of Migraine. Headache, 45, 144-152.

https://doi.org/10.1111/j.1526-4610.2005.05029.x 
20. International Headache Society (2018) The International Classification of Headache Disorders. 3rd Edition. http://ichd-3.org/

21. International Headache Society (1988) IHS Classification ICHD-II. http://www.ihs-klassifikation.de/en/01_einleitung/01_vorwort_auflage2/00.00.00_vorwortlaufl.html

22. Olesen, J. (2004) The International Classification of Headache Disorders. Cephalalgia, 24, 1-160.

23. Spierings, E.L. (2001) Migraine, Big and Small. Headache, 41, 918-922. https://doi.org/10.1046/j.1526-4610.2001.041009918.x

24. Olesen, J. and Lipton, R.B. (1994) Migraine Classification and Diagnosis. International Headache Society Criteria. Neurology, 44, S6-S10.

25. International Headache Society (2013) The International Classification of Headache Disorders. 3rd Edition. http://beta.ichd-3.org/

26. Shevel, E. and Shevel, D. (2014) The International Headache Society Classification of Migraine Headache-A Call for Substantiating Data. Journal of Biomedical Science and Engineering, 7, 112-114. https://doi.org/10.4236/jbise.2014.73015

27. Bakal, D.A. and Kaganov, J.A. (1977) Muscle Contraction and Migraine Headaches, Psycophysiologic Comparison. Headache, 17, 208-215. https://doi.org/10.1111/j.1526-4610.1977.hed1705208.x

28. Friedman, A.P., Von Storch, T.J. and Merritt, H.H. (1954) Migraine and Tension Headaches: A Clinical Study of Two Thousand Cases. Neurology, 4, 773-788. https://doi.org/10.1212/WNL.4.10.773

29. Olesen, J. (1978) Some Clinical Features of the Acute Migraine Attack. An Analysis of 750 Patients. Headache, 18, 268-271. https://doi.org/10.1111/j.1526-4610.1978.hed1805268.x 


\section{APPENDIX A: ABBREVIATIONS LIST}

\begin{tabular}{cc}
\hline AAN & $\begin{array}{c}\text { American Academy of Neurology } \\
\text { ACP-ASIM }\end{array}$ American College of Physicians-American Society of Internal Medicine \\
AHS & American Headache Society \\
BASH & British Association for the Study of Headache \\
CHS & Canadian Headache Society \\
EFNS & European Federation of Neurological Sciences \\
FLX & fluoxetine \\
ICHD & International Classification of Headache Disorders \\
IHS & International Headache Society \\
SNRI/s & Serotonin-norepinephrine Reuptake Inhibitor/s \\
SSRI/s & Selective Serotonin Reuptake Inhibitor/s \\
TCAs & tricyclic antidepressants \\
TTH/s & tension-type headache/s \\
\hline
\end{tabular}

